\title{
Variance maps as a novel tool for localizing regions of interest in imaging studies of individual differences
}

\author{
KAZUFUMI OMURA, ARTHUR ARON, and TURHAN CANLI \\ Stony Brook University, Stony Brook, New York
}

\begin{abstract}
Cognitive neuroimaging studies of individual differences seek to reveal brain mechanisms of cognition by associating intersubject variability in brain activation with other variables of interest, such as sex, personality trait, or mood state. The choice of a priori regions of interest (ROIs) raises problems, because the selection criterion is typically consistent activation across prior studies, suggesting little intersubject variability. Here, we introduce a novel approach for selecting regions that are defined on the basis of their variance characteristics, rather than on the basis of their location or because of theoretical expectations. These regions of variance (ROVs) constitute the search space with which to assess how much of the observed variance can be ascribed to specific variables of interest. We compare the ROI and ROV approaches by applying each to the same data set and suggest that the conjunction of both methods may yield the greatest likelihood of capturing the rich relation between brain and behavior, while limiting the search space for statistical analyses and minimizing false positive errors.
\end{abstract}

In functional neuroimaging studies in cognitive neuroscience, an individual-differences approach has increasingly been applied to reveal brain mechanisms of cognition. The aim of this approach is to associate individual differences in brain activation with meaningful determinants of variability, such as personality traits, sex, or genotype differences between individuals (Hamann \& Canli, 2004). One line of work has shown that brain activation in response to emotional stimuli consistently varies as a function of personality traits, such as extraversion or neuroticism (Amin, Constable, \& Canli, 2004; Canli, Amin, Haas, Omura, \& Constable, 2004; Canli, Sivers, Whitfield, Gotlib, \& Gabrieli, 2002; Canli et al. 2001).

Ideally, studies whose purpose is to associate individual differences in brain activation with other variables should be constrained by psychological theory and by the a priori selection of regions of interest (ROIs). However, ROIs are traditionally denoted as such because they have exhibited consistent activation across prior studies in which a similar task paradigm has been employed. The problem thus arises that these regions are least likely to exhibit much variability across individuals and, therefore, make poor choices for ROIs in imaging studies of individual differences.

In this article, we introduce an alternative methodology for defining ROIs in studies that apply an individualdifferences approach. This method is based on a proce-

This study was funded by SUNY-Stony Brook and by National Science Foundation Grant BCS-0224221. Correspondence concerning this article should be addressed to T. Canli, Department of Psychology, Stony Brook University, Stony Brook, NY 11794-2500 (e-mail:turhan. canli@sunysb.edu). dure that identifies regions of variance (ROVs) - that is, areas that display the most variability across subjects for a given within-subjects contrast. These ROVs are treated as ROIs and are then further investigated to assess whether particular variables of interest can explain the variance exhibited in these regions. We will briefly introduce the method for generating ROV maps and then will apply this method, using a previously published data set (Canli et al., 2004) with which a traditional ROI approach was used to evaluate the relation between brain reactivity to emotional stimuli and personality and mood state variables. By comparing the data obtained with these two approaches, we then will discuss the strengths and weaknesses of the ROV approach.

\section{METHOD}

\section{Development of a Variance Map}

We developed an ROV map by using the statistical parametric mapping software package SPM2 (Wellcome Department of Cognitive Neurology, London; http://www.fil.ion.ucl.ac.uk/spm). The data set that was the basis for our ROV map was obtained from an fMRI study (Canli et al., 2004) in which 12 subjects viewed alternating blocks of negative, neutral, or positive word stimuli during an emotional Stroop task (for details, see the fMRI Data Set section). Personality and mood state data were collected prior to scanning, to correlate brain activation to emotional, relative to neutral, word stimuli with these variables of interest. To quantify brain activation data, contrast images were calculated in SPM2 for each subject between negative-neutral and positive-neutral stimulus conditions. We used these contrast images from each subject to calculate a variance map across subjects. Thus, at the group level, each contrast was associated with its own between-subjects variance map.

The variance map was based on the ratio of between-subjects variance to within-subjects variance, which was calculated for each voxel with the SPM2 imcalc function. Between-subjects variance 
$\left(S_{\mathrm{B}}^{2}\right)$ can be calculated for each voxel from each subject's contrast image (con*img):

$$
S_{\mathrm{B}}^{2}=\frac{\frac{1}{N S u b j-1} \sum_{i=1}^{N S u b j}\left(\operatorname{con} * . i m g_{i}-\overline{\operatorname{con} * . i m g}\right)^{2}}{2} \times N S c a n .
$$

For each voxel, let NSubj denote the number of subjects and NScan represent the number of scans; con*.img is the voxel value from the $i$ th subject's contrast image, and $\overline{\text { con*.img }}$ is the mean voxel value across subjects from the contrast images. Contrast images are created when $t$ contrasts are calculated and correspond to the weighted sums of the beta weights, so that for a two-condition contrast, it represents a difference score between the voxel's activation values for the two conditions. The variance of difference scores, the numerator of Equation 1, must be divided by 2, because it is double the mean square for the interaction of subjects and conditions. (Note that if the contrast involves more than two conditions and it can be assumed that each condition has equal weight in the contrast, instead of dividing by two, one divides by the number of conditions in the contrast.) In order to make $S_{\mathrm{B}}^{2}$ comparable to the within-subjects variance as an estimate of the population of individual scans within conditions, one must multiply the numerator of Equation 1 by the number of scores in the condition (i.e., the number of scans, NScan).

The within-subjects variance $\left(S_{\mathrm{W}}^{2}\right)$ is calculated for each voxel from each subject's ResMS.img. ResMS.img represents images of the residual variance estimated that corresponds to the mean square errors $\left(M S_{\mathrm{e}}\right)$ in an analysis of variance (ANOVA). In SPM2, however, ResMS.img is saved as the sum of squares of errors $\left(S S_{\mathrm{e}}\right)$ of images, due to a bug in spm_spm.m, Version 2.66 (Meriaux, 2005; Nichols, 2005). This bug does not affect SPM2's variance estimate, because whenever ResMS.img is read, the scaling information is used (Nichols, 2005). (Note that SPM99 does not have this bug, so one does not have to divide the mean of ResMS.img by NScan-1 when using SPM99.) Within-subjects variance is calculated as

$$
S_{\mathrm{W}}^{2}=\frac{\frac{1}{N S u b j} \sum_{i=1}^{N S u b j} \operatorname{ResMS.img} g_{i}}{N S c a n-1} .
$$

For each voxel, let ResMS.img $g_{i}$ represent the $i$ th subject's residual image.

Taking into account the two variances, the image of the ratio of between-subjects variance to within-subjects variance is subjected to an $F$ test ( $F$ map) in order to create a mask image of regions where the between-subjects variance is significantly larger than the within-subjects variance:

$$
F=\frac{S_{\mathrm{B}}^{2}}{S_{\mathrm{W}}^{2}} .
$$

Degrees of freedom for $S_{\mathrm{B}}^{2}$ is denoted by NScan -1; degrees of freedom for $S_{\mathrm{W}}^{2}$ is denoted by $(N S c a n-1) \times(N S u b j \times N C o n d)$, where NCond is the number of conditions in the overall experiment (i.e., not just the number of conditions in the contrast being evaluated).

In order to exclude any white matter regions and to use this map in MNI (Montreal Neurological Institute) space, the gray matter template (gray.mnc) in SPM was multiplied, using the imcalc function in SPM2. According to this process, the dimension of the original variance map $(79 \times 95 \times 69)$ was transformed into MNI coordinates $(91 \times 109 \times 91)$.

The $F$ map was then subjected to a significance threshold of $p<$ .05 , using random field theory implemented in SPM2 (Worsley et al., 1996) and corrected for multiple comparisons.

\section{Integration With SPM}

The variance map procedure was implemented into the SPM2 software and integrated with an automated method for generating ROI masks according to the MNI space that was based on the Talairach Daemon database (Maldjian, Laurlenti, Kraft, \& Burdette,
2003) by modifying the spm_getSPM.m and wfu_spm_getSPM2.m files. We have made these files, along with user instructions, available for downloading from our Web site (http://www.psychology. sunysb.edu/tcanli-/). Briefly, the user is given the option of creating a variance map when pressing the "Results" button in SPM and is then prompted to select each subject's contrast image. The user is also given control over the minimum threshold of variance that determines which voxels will be included in the binary mask. In this article, we set the threshold to be $p<.05$, corrected. The program will then create a binary representation of the variance map, in which any voxels that have a value exceeding the minimum threshold will be given a value of 1 and all other voxels will be given a value of 0 . This binary representation of the variance map will then serve as a mask that constrains the search space within which subsequent analyses are conducted. The effect of this mask is that the available search space is dramatically reduced, minimizing the probability of false positive results, while maximizing power for obtaining results in the search space.

\section{fMRI Data Set}

Subjects. The data set we used was originally acquired by Canli et al. (2004). Twelve healthy right-handed subjects (6 males) were recruited. The subjects' mean age was 22.7 years $(S D=3.3$; range: 18-27). The subjects had no history of brain injury, reported no substance abuse within the past 6 months, were not on any moodaltering medication, and had no physical limitations that prohibited them from participating in an fMRI study.

Personality questionnaire. Prior to scanning, all the subjects completed the NEO Five-Factor Inventory (NEO-FFI; Costa \& McCrae, 1992), a 60-item self-report short form of the revised NEO Personality Inventory. The NEO-FFI covers each of the "Big 5" personality traits (neuroticism, extraversion, openness, agreeableness, and conscientiousness). Thus, sample scores for extraversion and neuroticism, the principal personality traits of interest in this study, were well within the range of the general population.

Experimental design. The subjects were placed into the scanner, where they viewed word stimuli displayed in blue, red, or green. They were asked to indicate, as quickly and accurately as possible, the color in which each word was printed by pressing a corresponding key on a button box. Word stimuli were selected from a stimulus library, the Affective Norms for English Words (ANEW) set (Bradley \& Lang, 1999). Alternating 30-sec blocks of positive, negative, and neutral word stimuli were shown six times per emotion condition. Each block contained 10 words, each of which was presented for $1,500 \mathrm{msec}$, followed by a 1,500 - $\mathrm{msec}$ presentation of a fixation cross. For each emotion condition, the first two blocks contained only novel words, and the remaining four blocks contained repeated presentations of these words. Thus, 20 unique words per emotion condition were shown three times each and were printed in a different color each time.

Imaging acquisition. Whole-brain imaging was performed on a 1.5-T GE Signa LX scanner. For structural whole-brain images, a three-dimensional high-resolution spoiled gradient (SPGR) scan and a T1 scan ( 35 slices, $3.5-\mathrm{mm}$ thickness; oriented parallel to the line between the anterior and the posterior commissure) were conducted. For functional images, T2*-weighted time series images depicting BOLD contrast (Ogawa, Lee, Kay, \& Tank, 1990) were acquired, using a gradient-echo echoplanar imaging sequence with a flip angle of $80^{\circ}$, repetition time $=3 \mathrm{sec}$, echo time $=45 \mathrm{msec}$, and field of view $=20 \times 20 \mathrm{~cm}$ (see Canli et al., 2004, for details).

Data analysis. Functional data were preprocessed and statistically analyzed using SPM2, with functional images realigned to the first in the time series, coregistered to the SPGR volume image, which was segmented and normalized to the gray matter template. Spatial transformations derived from normalizing the segmented gray matter were then applied to all functional volumes, which were then spatially smoothed with an 8-mm full-width-half-maximum isotropic Gaussian filter. 
Fixed-effects models were used at the individual subject level of analysis, and random-effects models (Holmes \& Friston, 1998) were used for group-level analyses. To evaluate the extent to which brain activation was correlated with individual differences in personality trait or mood state, scores for extraversion and positive mood were entered as regressors in a random-effects multiple regression model, using the positive-neutral contrast. A similar analysis had scores for neuroticism and negative mood entered as regressors, using the negative-neutral contrast. To control for the contribution of sex differences, sex was also entered in all analyses as a covariate.

Functional analyses were restricted to the a priori ROI. In the original study (Canli et al., 2004), this region was limited to the anterior cingulate $(\mathrm{AC})$, but in the present analysis, it was made up of all the voxels that were included in the variance map mask (see above). As in the original study, we applied a significance threshold of $p<.05$ (uncorrected), and an extent threshold of 10 voxels was applied to the search region.

\section{RESULTS}

\section{ROV Maps}

In our original study (Canli et al., 2004), the AC was the primary ROI. As is shown in Figure 1, the variance map approach identifies many more regions (ROVs) that make up the search space for subsequent correlational analyses (see the corresponding Table 1 or 2 for specific locations, coordinates, and cluster sizes). One first notices that the variance is not uniformly distributed across the brain but, rather, is localized within clusters, suggesting that it does not merely represent noise. A second observation is that several ROV clusters represent brain regions previously associated with emotional processes, such as the AC, the insula, and the precuneus (Allman, Hakeem, Erwin, Nimchinsky, \& Hof, 2001; Bush, Luu, \& Posner, 2000; Eugene et al., 2003; Liotti et al., 2000; Phillips, Drevets, Rauch, \& Lane, 2003; Teasdale et al., 1999). Third, the number and location of ROVs, although similar, are not identical across both maps, suggesting some task-relevant specificity in observed variance.

\section{Trait-State Correlations Detected With the ROI Versus the ROV Approach}

The variance maps were used as masks to define the search space for subsequent multiple regression analyses. Multiple regression analyses were conducted to identify regions where greater activation to emotional, relative to neutral, words correlated with either personality
A

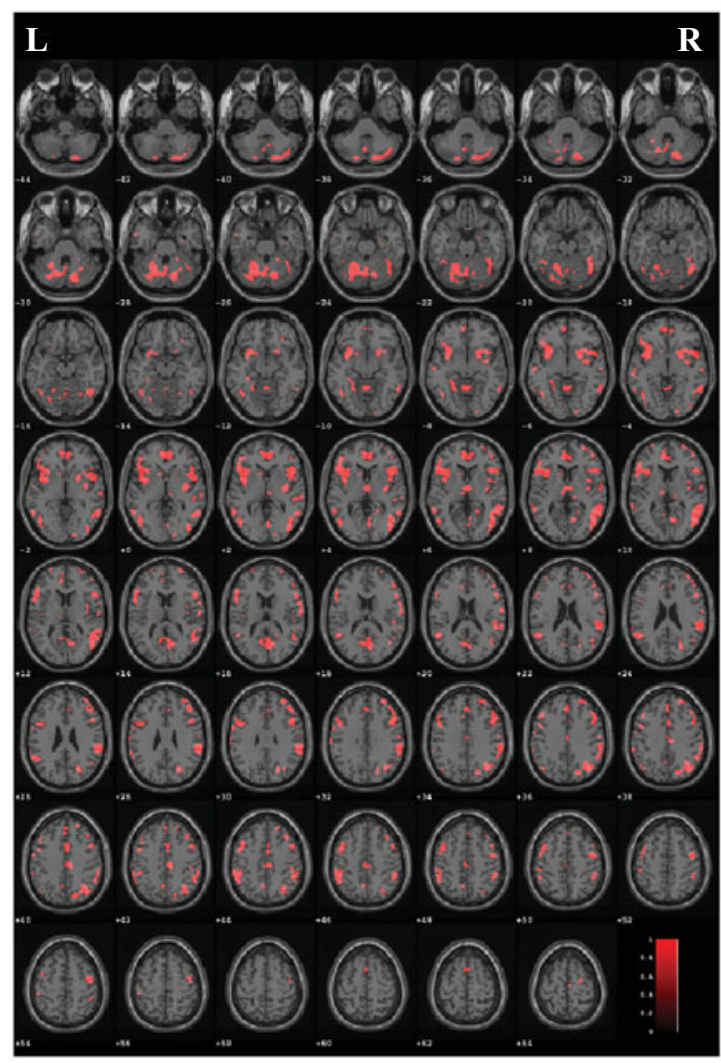

B

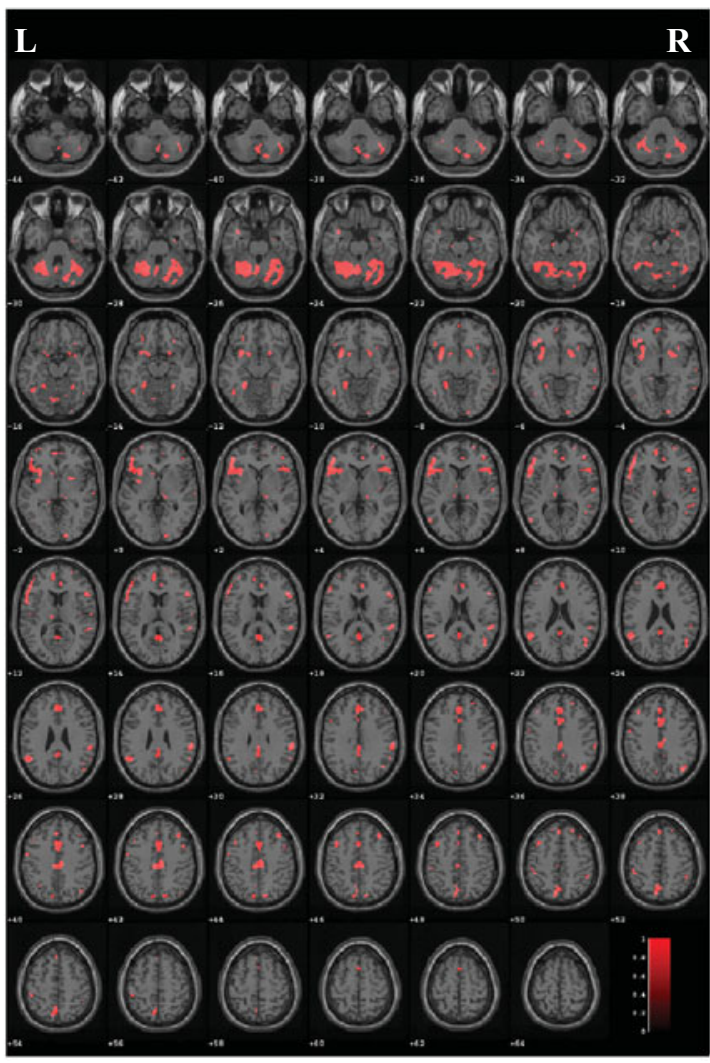

Figure 1. Variance maps based on positive-neutral (A) and negative-neutral (B) contrasts. Both maps were overlaid onto a standardized structural T1-weighted MRI template with axial orientation in each contrast. Axial images are representative slices displayed in $2-\mathrm{mm}$ increments along the $z$-axis $(z=-44$ to 64$)$. Regions of variance are indicated in red. $L$ indicates left hemisphere, and $R$ indicates right hemisphere. 
Table 1

Anatomical Locations and Stereotaxic Coordinates of Regions of Variance Revealed by Positive-Neutral Contrast

\begin{tabular}{|c|c|c|c|c|c|c|}
\hline \multirow[b]{2}{*}{ Brain Region } & \multirow[b]{2}{*}{ Hemisphere } & \multirow[b]{2}{*}{ Anatomical Location } & \multicolumn{3}{|c|}{ MNI Coordinates } & \multirow[b]{2}{*}{ Cluster Size } \\
\hline & & & $x$ & $y$ & $z$ & \\
\hline \multirow[t]{15}{*}{ Frontal } & Left & IFG, extending into insula & -54 & 16 & 34 & 2,114 \\
\hline & & Insula & -40 & -16 & 12 & 11 \\
\hline & & MedOFG, extending into $\mathrm{AC}$ & 28 & 56 & 28 & 594 \\
\hline & & PreCG & -58 & 6 & 30 & 36 \\
\hline & & & -58 & 26 & 40 & 13 \\
\hline & & SFG & -30 & 62 & 10 & 19 \\
\hline & & $\mathrm{AC}$ & 28 & 32 & 22 & 12 \\
\hline & Right & IFG/MFG & 38 & 48 & 30 & 2,147 \\
\hline & & IFG & 46 & 46 & 12 & 70 \\
\hline & & & 32 & 30 & -14 & 13 \\
\hline & & MedSFG, extending into AC & 4 & 42 & 40 & 141 \\
\hline & & PreCG & 36 & 26 & 54 & 133 \\
\hline & & & 22 & 28 & 64 & 15 \\
\hline & & $\mathrm{AC}$ & 4 & 4 & 44 & 87 \\
\hline & & SMA & 6 & 8 & 62 & 22 \\
\hline \multirow[t]{8}{*}{ Temporal } & Left & MTG & -62 & -48 & 2 & 159 \\
\hline & & & -50 & 2 & -30 & 16 \\
\hline & & FG & -44 & -48 & -22 & 52 \\
\hline & & STG & -60 & -26 & 22 & 47 \\
\hline & Right & PostMTG & 52 & -72 & 12 & 1,051 \\
\hline & & FG & 38 & -60 & -18 & 345 \\
\hline & & & 24 & -62 & -16 & 19 \\
\hline & & MTG & 48 & -30 & 2 & 104 \\
\hline \multirow[t]{9}{*}{ Parietal } & Left & PostCG, extending into SMG & -46 & -30 & 58 & 467 \\
\hline & & PostCG & -64 & -18 & 32 & 28 \\
\hline & & $\mathrm{PC}$ & 0 & -12 & 36 & 185 \\
\hline & & Precuneus & 24 & -58 & 38 & 54 \\
\hline & & AG & -40 & -74 & 36 & 33 \\
\hline & Right & SMG & 58 & -42 & 42 & 807 \\
\hline & & AG & 30 & -70 & 48 & 616 \\
\hline & & PostCG & 64 & 26 & 10 & 172 \\
\hline & & Precuneus & 24 & -50 & 4 & 25 \\
\hline \multirow[t]{2}{*}{ Occipital } & Right & CalcS & 2 & -72 & 4 & 417 \\
\hline & & & 14 & -96 & 0 & 78 \\
\hline Basal ganglia & Left & Caudate & -10 & 12 & 24 & 51 \\
\hline \multirow{2}{*}{ Thalamus } & Right & Thalamus & 4 & -20 & 2 & 153 \\
\hline & & & 12 & -20 & 10 & 13 \\
\hline \multirow[t]{6}{*}{ Cerebellum } & Left & Cerebellum & -16 & -78 & -38 & 97 \\
\hline & Right & Cerebellum & 42 & -64 & -38 & 2,255 \\
\hline & & & 26 & -58 & -50 & 36 \\
\hline & & & 28 & -88 & -22 & 14 \\
\hline & & & 24 & -44 & -30 & 14 \\
\hline & & Vermis & 4 & -54 & -14 & 152 \\
\hline
\end{tabular}

Note-IFG, inferior frontal gyrus; MedOFG, medial part of orbitofrontal gyrus; AC, anterior cingulate; PreCG, precentral gyrus; SFG, superior frontal gyrus; MFG, middle frontal gyrus; MedSFG, medial part of superior frontal gyrus; SMA, supplementary motor area; MTG, middle temporal gyrus; FG, fusiform gyrus; STG, superior temporal gyrus; PostMTG, posterior part of middle temporal gyrus; AG, angular gyrus; SMG, supramarginal gyrus; PostCG, postcentral gyrus; CalcS, calcarine sulcus.

traits (controlled for mood state and for sex) or with mood state (controlled for personality trait and for sex). In Figures 2 and 3, the results for positive and for negative stimuli, respectively, are compared when the search space is defined by a single ROI (the AC) or by the ROV approach. Table 3 lists the regions where the ROV approach identified significant correlations. Using the traditional ROI approach, we had previously reported that $\mathrm{AC}$ activation to positive, relative to neutral, stimuli varies as a function of extraversion, but not of positive mood (Canli et al., 2004). This finding was replicated with the ROV approach (see Table 3), which also repli- cated another observation made with the ROI approach, that $\mathrm{AC}$ activation to negative, relative to neutral, stimuli varies as a function of negative mood, but not of neuroticism (Canli et al., 2004). Thus, there is some correspondence between both approaches in identifying significant brain-behavior correlations.

However, the ROV approach offers more opportunities to identify significant correlations, because it includes a larger set of ROIs. For example, for positive stimuli (Figure 2), the ROI approach did not reveal any regions where brain activation to positive, relative to neutral, words was associated with higher positive mood 
Table 2

Anatomical Locations and Stereotaxic Coordinates of Regions of Variance Revealed by Negative-Neutral Contrast

\begin{tabular}{|c|c|c|c|c|c|c|}
\hline \multirow[b]{2}{*}{ Brain Region } & \multirow[b]{2}{*}{ Hemisphere } & \multirow[b]{2}{*}{ Anatomical Location } & \multicolumn{3}{|c|}{ MNI Coordinates } & \multirow[b]{2}{*}{ Cluster Siz } \\
\hline & & & $x$ & $y$ & $z$ & \\
\hline \multirow[t]{22}{*}{ Frontal } & Left & IFG, extending into insula & -42 & 36 & 6 & 1,247 \\
\hline & & $\mathrm{AC}$ & -4 & 50 & 12 & 81 \\
\hline & & MFG & -40 & 16 & 44 & 80 \\
\hline & & & -36 & 50 & 14 & 13 \\
\hline & & MedOFG, extending into $\mathrm{AC}$ & -4 & 46 & -4 & 39 \\
\hline & & PreCG & -56 & -6 & 38 & 32 \\
\hline & & OFG & -28 & 30 & -16 & 20 \\
\hline & & SFG/MFG & -28 & 60 & 0 & 14 \\
\hline & Right & $\mathrm{AC}$ & 8 & 42 & 2 & 482 \\
\hline & & & 4 & 4 & 42 & 278 \\
\hline & & & 10 & 48 & 0 & 13 \\
\hline & & IFG, extending into insula & 38 & 20 & 8 & 278 \\
\hline & & IFG & 44 & 38 & 4 & 44 \\
\hline & & Insula & 36 & 20 & -6 & 29 \\
\hline & & & 40 & -10 & 4 & 10 \\
\hline & & MFG & 32 & 30 & 46 & 68 \\
\hline & & & 26 & 44 & 34 & 12 \\
\hline & & SFG/MFG & 26 & 58 & 14 & 34 \\
\hline & & SFG & 24 & 34 & 50 & 13 \\
\hline & & SMA & 4 & 10 & 62 & 29 \\
\hline & & PreCG & 46 & 12 & 40 & 24 \\
\hline & & OFG & 32 & 30 & -14 & 12 \\
\hline \multirow[t]{7}{*}{ Temporal } & Left & Temporal pole, extending into amygdala & -36 & 10 & -26 & 40 \\
\hline & & PostMTG & -52 & -66 & 4 & 20 \\
\hline & Right & Amygdala, extending into putamen & 32 & 4 & -16 & 221 \\
\hline & & STG & 62 & -16 & 6 & 40 \\
\hline & & Hippocampus, extending into amygdala & 30 & -8 & -28 & 34 \\
\hline & & PostMTG & 60 & -54 & -6 & 19 \\
\hline & & MTG & 26 & 44 & 34 & 12 \\
\hline \multirow[t]{10}{*}{ Parietal } & Left & Cuneus, extending into precuneus & -6 & -74 & 30 & 264 \\
\hline & & AG & -48 & -58 & 24 & 190 \\
\hline & & PostCG & -48 & -32 & 54 & 43 \\
\hline & Right & $\mathrm{PC}$ & 10 & -18 & 44 & 638 \\
\hline & & SMG & 60 & -26 & 28 & 265 \\
\hline & & & 58 & -52 & 26 & 21 \\
\hline & & AG & 46 & -72 & 28 & 80 \\
\hline & & & 44 & -70 & 32 & 73 \\
\hline & & Precuneus & 10 & -78 & 42 & 41 \\
\hline & & IPL & 48 & -42 & 54 & 20 \\
\hline Occipital & Right & CalcS & 18 & -96 & -4 & 78 \\
\hline \multirow[t]{2}{*}{ Thalamus } & Left & Thalamus & -8 & -16 & 10 & 35 \\
\hline & & Thalamus & 16 & -26 & 4 & 31 \\
\hline \multirow[t]{3}{*}{ Cerebellum } & Left & Cerebellum, extending into FG & -16 & -72 & -22 & 3,482 \\
\hline & & Cerebellum & -12 & -50 & -50 & 113 \\
\hline & Right & Cerebellum & 26 & -48 & -50 & 75 \\
\hline
\end{tabular}

Note-IFG, inferior frontal gyrus; AC, anterior cingulate; MFG, middle frontal gyrus; MedOFG, medial part of orbitofrontal gyrus; PreCG, precentral gyrus; OFG, orbitofrontal gyrus; SFG, superior frontal gyrus; SMA, supplementary motor area; PostMTG, posterior part of middle temporal gyrus; STG, superior temporal gyrus; MTG, middle temporal gyrus; AG, angular gyrus; PostCG, postcentral gyrus; PC, posterior cingulate; SMG, supramarginal gyrus; IPL, inferior parietal lobule; CalcS, calcarine sulcus; FG, fusiform gyrus.

scores (controlling for extraversion and sex). With the ROV approach, large clusters in the posterior part of the right middle temporal gyrus and the right middle frontal gyrus were identified (Table 3 ). Some clusters were also found in the left cerebellum, the left superior frontal gyrus, and the right anterior gyrus. Whereas the ROI approach was limited to documenting a correlation between extraversion (controlling for positive mood and sex) and AC activation to positive, relative to neutral, words, the ROV approach added the left medial orbito- frontal gyrus and the bilateral insula to the list of regions. Thus, the benefit of the ROV approach is that it detects regions that are significantly correlated with a variable of interest that would otherwise be missed with the ROI approach. It is interesting to note, however, that a significant correlation that was observed with the ROI approach was not observed with the ROV approach: The ROI approach revealed a cluster located within the left AC (31 voxels in size; MNI coordinates, $-14,23,26$; $p=.009)$, where greater activation was significantly 
Table 3

Stereotaxic Coordinates of Significant Activations Revealed by Each Contrast

\begin{tabular}{|c|c|c|c|c|c|c|c|c|}
\hline \multirow[b]{2}{*}{ Contrast } & \multirow[b]{2}{*}{ Hemisphere } & \multirow[b]{2}{*}{ Anatomical Location } & \multicolumn{3}{|c|}{ MNI Coordinates } & \multirow[b]{2}{*}{$t$ Score } & \multirow[b]{2}{*}{$p$ Value } & \multirow{2}{*}{$\begin{array}{l}\text { Cluster } \\
\text { Size }\end{array}$} \\
\hline & & & $x$ & $y$ & $z$ & & & \\
\hline \multicolumn{9}{|l|}{ Positive-Neutral } \\
\hline \multirow{6}{*}{$\begin{array}{l}\text { Correlation with positive mood } \\
\quad \text { (controlled for extraversion and sex) }\end{array}$} & Left & SFG & -30 & 62 & 10 & 3.89 & .002 & 19 \\
\hline & & Cerebellum & -30 & -78 & -24 & 2.99 & .009 & 52 \\
\hline & Right & PostMTG & 52 & -72 & 12 & 3.65 & .003 & 183 \\
\hline & & MFG & 38 & 48 & 30 & 3.14 & .007 & 129 \\
\hline & & & 40 & 52 & 2 & 2.32 & .024 & 10 \\
\hline & & $\mathrm{AG}$ & 32 & -60 & 34 & 2.54 & .017 & 21 \\
\hline \multirow{9}{*}{$\begin{array}{l}\text { Correlation with extraversion } \\
\text { (controlled for positive mood and sex) }\end{array}$} & Left & MedOFG & -8 & 56 & -8 & 3.72 & .003 & 40 \\
\hline & & IFG & -50 & 32 & 2 & 3.04 & .008 & 119 \\
\hline & & Insula & -44 & 0 & 0 & 2.67 & .014 & 66 \\
\hline & & & -38 & 18 & -6 & 2.47 & .019 & 35 \\
\hline & Right & LG & 6 & -72 & 2 & 4.29 & .001 & 56 \\
\hline & & $\mathrm{AC}$ & 6 & 48 & 6 & 2.92 & .010 & 57 \\
\hline & & Insula & 40 & 12 & -8 & 2.79 & .012 & 36 \\
\hline & & & 40 & -4 & -6 & 2.42 & .021 & 16 \\
\hline & & AG & 48 & -62 & 24 & 2.39 & .022 & 11 \\
\hline \multicolumn{9}{|l|}{ Negative-Neutral } \\
\hline \multirow{20}{*}{$\begin{array}{l}\text { Correlation with negative mood } \\
\text { (controlled for neuroticism and sex) }\end{array}$} & Left & Cerebellum, extending into FG & -20 & -54 & -18 & 3.88 & .002 & 669 \\
\hline & & & -10 & -70 & -18 & 2.53 & .017 & 18 \\
\hline & & PostMTG & -52 & -66 & 4 & 2.96 & .009 & 20 \\
\hline & & IFG & -44 & 32 & 10 & 2.60 & .016 & 30 \\
\hline & & Putamen, extending into amygdala & -36 & 0 & -10 & 2.59 & .016 & 46 \\
\hline & & Precuneus & -4 & -52 & 18 & 2.49 & .018 & 55 \\
\hline & & Vermis & -2 & -72 & -40 & 2.39 & .022 & 18 \\
\hline & & PostITG & -46 & -62 & -12 & 2.17 & .031 & 13 \\
\hline & & MedOFG, extending into $\mathrm{AC}$ & -4 & 46 & -4 & 2.10 & .034 & 10 \\
\hline & Right & Cerebellum, extending into FG & 36 & -64 & -20 & 3.28 & .006 & 300 \\
\hline & & Cerebellum & 12 & -70 & -46 & 2.99 & .009 & 35 \\
\hline & & & 14 & -68 & -26 & 2.22 & .028 & 13 \\
\hline & & & 26 & -48 & -48 & 2.02 & .038 & 11 \\
\hline & & $\mathrm{PC}$ & 10 & -18 & 44 & 3.12 & .007 & 139 \\
\hline & & STG & 62 & -16 & 6 & 2.87 & .010 & 35 \\
\hline & & LG & 24 & -88 & -18 & 2.71 & .013 & 12 \\
\hline & & Amygdala & 32 & 4 & -16 & 2.57 & .016 & 26 \\
\hline & & Thalamus & 10 & -24 & 2 & 2.35 & .023 & 23 \\
\hline & & $\mathrm{AC}$ & 4 & 4 & 38 & 2.30 & .025 & 11 \\
\hline & & CalcS & 16 & -96 & -4 & 2.01 & .039 & 28 \\
\hline \multirow{2}{*}{$\begin{array}{l}\text { Correlation with neuroticism } \\
\text { (controlled for negative mood and sex) }\end{array}$} & Left & SFG/MFG & -28 & 60 & 0 & 2.35 & .023 & 10 \\
\hline & Right & Hippocampus, extending into amygdala & 28 & -6 & -26 & 2.60 & .016 & 22 \\
\hline
\end{tabular}

Note-Significance level was set at $p<.05$ (uncorrected for multiple comparisons), extent threshold $=10$ voxels. SFG, superior frontal gyrus; PostMTG, posterior part of middle temporal gyrus; MFG, middle frontal gyrus; AG, angular gyrus; MedOFG, medial part of orbitofrontal gyrus; IFG, inferior frontal gyrus; LG, lingual gyrus; AC, anterior cingulate; FG, fusiform gyrus; PostITG, posterior part of inferior temporal gyrus; PC, posterior cingulate; STG, superior temporal gyrus; CalcS, calcarine sulcus.

correlated with higher extraversion scores (controlling for positive mood and sex), whereas the ROV approach failed to detect this cluster. The reason is that the inclusion criterion for voxels in the variance map was set to a minimum of $p<.05$ (corrected) and that the range of beta values from the subjects' contrast files in the left $\mathrm{AC}$ cluster was below this minimum threshold. Thus, the specific region that was included in the search space defined by the ROI approach was not included in the ROV map.

For negative stimuli (Figure 3), the ROI approach did not reveal any regions where brain activation to negative, relative to neutral, words was associated with higher neuroticism scores (controlling for negative mood and sex). The ROV approach identified the left middle frontal gyrus and the right amygdala as regions where such correlations are significant. With ROVs, several regions were also identified where greater activation to negative, relative to neutral, words was correlated with higher negative mood scores (controlling for neuroticism and sex), including the bilateral cerebellum, the left precuneus, the bilateral amygdala, and the posterior cingulate.

One striking finding is that the ROV approach identified areas within the cerebellum as varying in activation to emotional stimuli as a function of mood. In the original study (Canli et al., 2004), the cerebellum was not included in the analysis as an a priori ROI, and no clusters within the cerebellum were detected when a post hoc analysis (which applied a more stringent statistical threshold criterion) was conducted. The variance detected in 


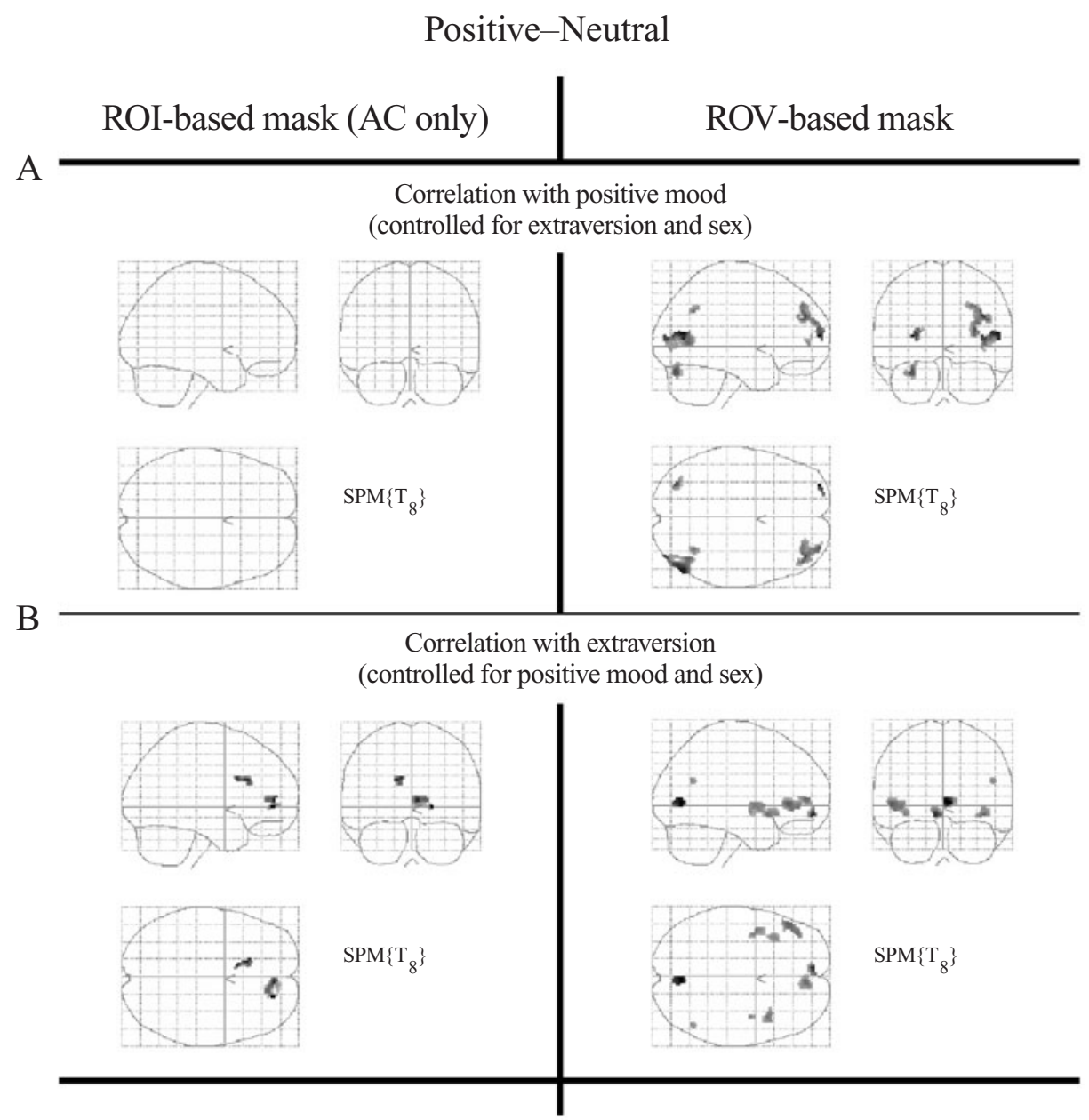

\begin{abstract}
Figure 2. Sagittal, coronal, and axial views of significant correlation clusters located within a predetermined search space, as defined using the region-of-interest (ROI; left panels) and region-ofvariance (ROV; right panels) approaches. In the left panels, search space is defined solely by the anterior cingulate (AC); in the right panels, search space is defined by the ROV variance map. Panel $A$ shows regions where increased activation in the $\mathrm{AC}$ and in the variance map to positive words was significantly correlated with mood state (controlling for personality traits and for sex). Panel B shows regions where increased activation in the $\mathrm{AC}$ and in the variance map to positive words was significantly correlated with personality (controlling for mood state and for sex).
\end{abstract}

this region is not likely to reflect mere physiological noise, because the variance map was calculated in consideration of within-subjects variance.

\section{DISCUSSION}

In this article, we introduced the concept of a variance map in order to address the problem of defining ROIs for neuroimaging studies, using an individual-differences approach. The traditional method of choosing a priori ROIs relies on selecting brain regions that have been noted in the previous literature. These regions typically exhibit consistent activation across studies in which similar paradigms were used and are therefore believed to be associated with the task of interest. However, studies that are designed to investigate individual differences seek to identify regions that exhibit maximal variability across subjects, rather than consistent activation. Indeed, it is conventional in other research contexts, such as meta-analysis and multilevel modeling, to exclude any variable that does not have significant variance from correlational analyses (Kreft \& de Leeuw, 1998; Lipsey \& Wilson, 2001).

We therefore introduced the ROV approach, which identifies areas of maximal variance for a given data set. Note that the selection of ROVs is devoid of any theoretical assumptions or biases about the neural substrate and its relation to the cognitive process under study. In 


\title{
Negative-Neutral
}

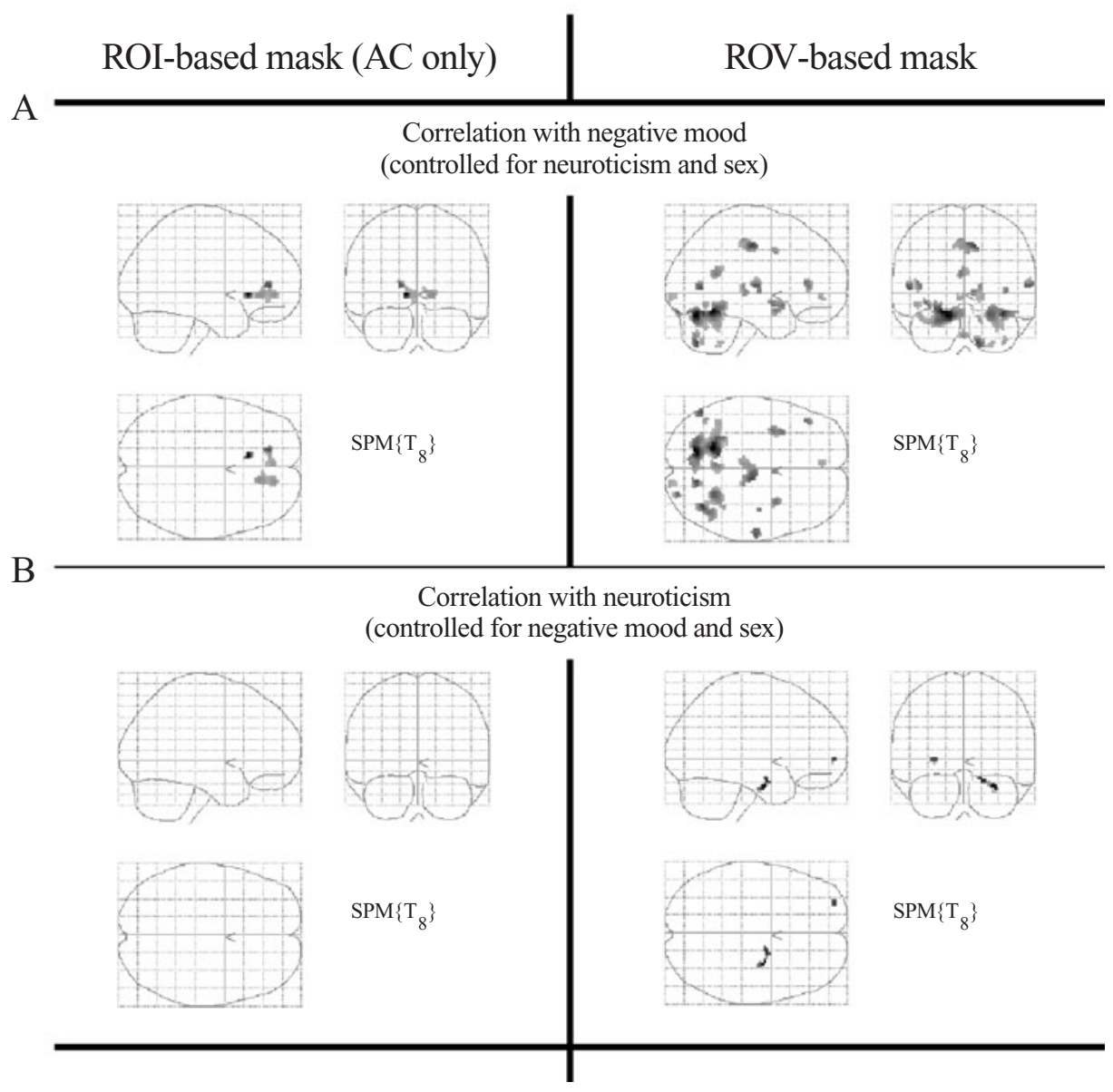

\begin{abstract}
Figure 3. Sagittal, coronal, and axial views of significant correlation clusters located within a predetermined search space, as defined using the region-of-interest (ROI; left panels) and region-ofvariance (ROV; right panels) approaches. In the left panels, search space is defined solely by the anterior cingulate (AC); in the right panels, search space is defined by the ROV variance map. Panel $A$ shows regions where increased activation in the $\mathrm{AC}$ and in the variance map to negative words was significantly correlated with mood state (controlling for personality traits and for sex). Panel B shows regions where increased activation in the $\mathrm{AC}$ and in the variance map to negative words was significantly correlated with personality (controlling for mood state and for sex).
\end{abstract}

contrast, the selection of ROIs typically involves a considerable number of assumptions about the cognitive functions that they are believed to play a role in. Thus, from the outset, ROIs and ROVs represent very different approaches to the study of brain function.

When we constructed variance maps based on a data set of brain activations to emotional, relative to neutral, word stimuli, we found that variance was not diffusely distributed (as one might expect if it were noise) but, rather, clustered around specific neural regions, including some regions known to play a role in emotional processes. We also noted that these clusters were located in different regions for different contrasts, suggesting some degree of stimulus specificity.

We then used the variance maps as masks to define the space within which we conducted subsequent multiple regression analyses. If one compares the results when this space was defined by the traditional ROI approach (which limited the analysis to one region, the $\mathrm{AC}$ ) with those of the ROV approach, several observations can be made. First, the ROV approach can replicate observations made with the ROI approach. For example, the association between AC activation to positive word stimuli and extraversion can be observed with either method. However, the ROI approach is usually limited to one or very few a priori ROIs, whereas the ROV approach will include any region that has significant variance. It is therefore not surprising that more regions can be identified that exhibit brain-behavior correlations of interest with this methodology, as was indeed demonstrated here. The most striking example of this was the observation that activation in the cerebellum varies as a function of 
positive mood in response to positive words and as a function of negative mood in response to negative words. This region was not detected with the ROI approach, since neither was it an a priori region nor did it achieve sufficient statistical significance to survive a more stringent statistical threshold for a post hoc analysis. Nonetheless, a role of the cerebellum in emotional processing has been proposed (Schmahmann \& Sherman, 1998) and deserves to be followed up in future work.

The ROV approach does, on occasion, miss interesting brain-behavior correlations. For example, an association between extraversion and $\mathrm{AC}$ activation to positive stimuli was observed within the left AC with the ROI approach, but not with the ROV approach. This was due to the fact that, even though the correlation was highly significant, the range of values that contributed to this correlation was relatively narrow (i.e., between-subjects variance was low). Because we had set the minimum criterion for the voxels to be included in the variance map to be $p<.05$, corrected, this cluster did not reach criterion and was, therefore, not included in the ROV search space.

The ROV approach not only is a useful tool for identifying regions that exhibit significant correlations with variables of interest, but also identifies regions where these variables are not sufficient to explain the observed variance. A comparison of Tables 1 and 2 with Table 3 readily shows that some regions exhibit variance that is not captured by the trait or state variables used here. For example, the variance map identifies a cluster of 54 voxels located in the left precuneus. Yet a multiple regression analysis in SPM of mood state and personality trait scores fails to detect a significant correlation in this region. When we extracted the average signal from this cluster and conducted a partial regression analysis in SPSS, with mood state, personality trait, and sex entered as variables of interest (data not shown), the proportion of variance $\left(R^{2}\right)$ accounted for by the total of these three variables was only .03. Given that the variance map identifies a prominent cluster in a region previously associated with emotion-related processes that cannot be satisfactorily explained by variability in personality trait, mood state, or sex, follow-up work should continue to seek variables that may better explain this variance.

Thus, the ROV approach identifies regions that exhibit significant between-subjects activation differences, regardless of any specific variable of interest. It identifies regions with significant between-subjects variance that are to be explained by individual-differences variables, as well as regions where there is insufficient variance to have high power to detect correlations with individualdifferences variables of interest. This second feature may be useful when an ROI-based or whole-brain-based analysis fails to show a significant correlation. In this case, it would be unclear whether this null result is due to the psychometric limitations of the variable being studied (i.e., insufficient between-subjects variance) or to conceptually incorrect assumptions (i.e., there is betweensubjects variance, but the variable of interest does not explain it). The ROV map could clarify whether there is sufficient variance to exclude psychometric problems as an explanation for the null result. Furthermore, the areas not included in the ROV map are of some theoretical interest, because they represent regions with high levels of similarity of response across subjects and, thus, may represent invariant species-wide adaptations.

In conclusion, we suggest that the ROI and the ROV methods are best thought of as complementary, rather than competing, approaches with which to identify regions where individual differences of interest are associated with variation in brain activity levels. The standard ROI approach is useful, because some areas that exhibit significant correlations may not exhibit sufficient variance to survive multiple-comparison corrections. The ROV approach, on the other hand, has the benefit that between-subjects variance allows investigators to localize regions that have not consistently been noted in prior published work and to identify regions of significant between-subjects variance to be explained by individualdifferences variables (whether these variables have been considered by the investigators a priori or remain to be discovered) and helps them determine whether observed null correlations may be due to psychometric or substantive limitations (i.e., a lack of significant betweensubjects variance). The combination of both approaches maximizes the opportunity to reveal the neural basis of complex variable behaviors.

\section{REFERENCES}

Allman, J. M., Hakeem, A., Erwin, J. M., Nimchinsky, E., \& Hof, P. (2001). The anterior cingulate cortex. The evolution of an interface between emotion and cognition. In A. R. Damasio, A. Harrington, J. Kasan, B. S. McEwen, H. Moss, \& R. Shailah (Eds.), Unity of knowledge: The convergence of natural and human science (Annals of the New York Academy of Sciences, Vol. 935, pp. 107-117). New York: New York Academy of Sciences.

Amin, Z., Constable, R. T., \& Canli, T. (2004). Attentional bias for valenced stimuli as a function of personality in the dot-probe task. Journal of Research in Personality, 38, 15-23.

Bradley, M. M., \& Lang, P. J. (1999). Affective norms for English words (ANEW): Instruction manual and affective ratings (Tech. Rep. C-1). Gainesville: University of Florida, Center for Research in Psychophysiology.

Bush, G., LuU, P., \& Posner, M. I. (2000). Cognitive and emotional influences in anterior cingulate cortex. Trends in Cognitive Sciences, $\mathbf{4}$, 215-222.

Canli, T., Amin, Z., Haas, B., Omura, K., \& Constable, R. T. (2004). A double dissociation between mood states and personality traits in the anterior cingulate. Behavioral Neuroscience, 118, 897-904.

Canli, T., Sivers, H., Whitfield, S. L., Gotlib, I. H., \& Gabrieli, J.D.E. (2002). Amygdala response to happy faces as a function of extraversion. Science, 296, 2191.

Canli, T., Zhao, Z., Desmond, J. E., Kang, E., Gross, J., \& Gabrieli, J.D.E. (2001). An fMRI study of personality influences on brain reactivity to emotional stimuli. Behavioral Neuroscience, 115, 3342.

Costa, P. T., \& McCrae, R. R. (1992). Professional manual of the Revised NEO Personality Inventory and NEO Five-Factor Inventory. Odessa, FL: PAR Inc.

Eugene, F., Levesque, J., Mensour, B., Leroux, J. M., Beaudoin, G., Bourgouin, P., \& Beauregard, M. (2003). The impact of individual differences on the neural circuitry underlying sadness. NeuroImage, 19, 354-364.

HAMANN, S., \& CANLI, T. (2004). Individual differences in emotion processing. Current Opinion in Neurobiology, 14, 233-238. 
Holmes, A. P., \& Friston, K. J. (1998). Generalisability, random effects and population inference [Abstract]. NeuroImage, 7, S754.

KREFT, I., \& DE LeEUW, J. (1998). Introduction to multilevel modeling. London: Sage.

Liotti, M., Mayberg, H. S., Brannan, S. K., McGinnis, S., JerABEK, P., \& FoX, P. T. (2000). Differential limbic-cortical correlates of sadness and anxiety in healthy subjects: Implications for affective disorders. Biological Psychiatry, 48, 30-42.

Lipsey, M. W., \& WiLson, D. B. (2001). Practical meta-analysis. Thousand Oaks, CA: Sage.

Maldjian, J. A., Laurienti, P. J., Kraft, R. A., \& Burdette, J. H. (2003). An automated method for neuroanatomic and cytoarchitectonic atlas-based interrogation of fMRI data sets. NeuroImage, 19, 1233-1239.

Meriaux, S. (2005, March 25). Problem using 'ResMS.img' with SPM2. http://www.jiscmail.ac.uk/cgi-bin/webadmin?A2=ind0503\&L= $\mathrm{spm} \& \mathrm{O}=\mathrm{D} \& \mathrm{~F}=\& \mathrm{~S}=\& \mathrm{P}=43984$.

Nichols, T. (2005, March 25). Re: Problem using 'ResMS.img' with SPM2. http://www.jiscmail.ac.uk/cgi-bin/webadmin?A2=ind0503\&L= spm\&O $=\mathrm{D} \& \mathrm{~F}=\& \mathrm{~S}=\& \mathrm{P}=44436$.
Ogawa, S., Lee, T. M., Kay, A. R., \& TanK, D. W. (1990). Brain magnetic resonance imaging with contrast dependent on blood oxygenation. Proceedings of the National Academy of Sciences, 87, 98689872.

Phillips, M. L., Drevets, W. C., Rauch, S. L., \& Lane, R. (2003). Neurobiology of emotion perception: I. The neural basis of normal emotion perception. Biological Psychiatry, 54, 504-514.

Schmahmann, J. D., \& Sherman, J. C. (1998). The cerebellar cognitive affective syndrome. Brain, 121 (Pt. 4), 561-579

Teasdale, J. D., Howard, R. J., Cox, S. G., Ha, Y., Brammer, M. J. Williams, S. C., \& Checkley, S. A. (1999). Functional MRI study of the cognitive generation of affect. American Journal of Psychiatry, 156, 209-215.

Worsley, K., Marrett, S., Neelin, P., Vandal, A., Friston, K., \& Evans, A. (1996). A unified statistical approach for determining significant signals in images of cerebral activation. Human Brain Mapping, 4, 58-73.

(Manuscript received September 15, 2004; revision accepted for publication April 18, 2005.) 